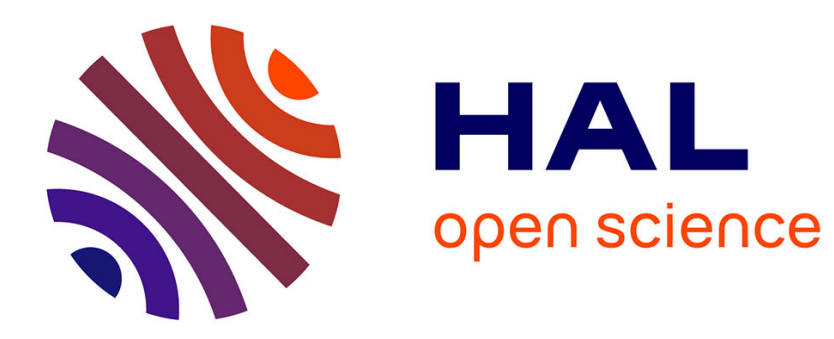

\title{
L'intégration du développement durable dans le financement bancaire aux entreprises
}

Vincent Maymo, Valérie Pallas

\section{To cite this version:}

Vincent Maymo, Valérie Pallas. L'intégration du développement durable dans le financement bancaire aux entreprises. La Revue des Sciences de Gestion, 2011, 10.3917/rsg.247.0139 hal-02952290

\author{
HAL Id: hal-02952290 \\ https://hal.science/hal-02952290
}

Submitted on 29 Sep 2020

HAL is a multi-disciplinary open access archive for the deposit and dissemination of scientific research documents, whether they are published or not. The documents may come from teaching and research institutions in France or abroad, or from public or private research centers.
L'archive ouverte pluridisciplinaire HAL, est destinée au dépôt et à la diffusion de documents scientifiques de niveau recherche, publiés ou non, émanant des établissements d'enseignement et de recherche français ou étrangers, des laboratoires publics ou privés. 


\title{
L'intégration du développement durable dans le financement bancaire aux entreprises
}

\author{
par Vincent Maymo et Valérie Pallas Saltiel
}

\author{
Vincent MAYMO \\ Maître de conférences \\ IRGO /EFFI - Université de Bordeaux \\ France
}

Valérie PALLAS SALTIEL

Maître de conférences

Institut de Recherche en Gestion - UPEC

France 


\title{
Résumé \\ L'intégration du développement durable dans le financement bancaire aux entreprises par Vincent Maymo et Valérie Pallas Saltiel
}

Cet article étudie les stratégies d'intégration du développement durable (et notamment de la dimension environnementale) dans les pratiques bancaires de financement des entreprises. Au-delà d'une étude des motivations, il propose, en croisant la littérature en management environnemental avec les théories institutionnelles, une grille de lecture des processus d'institutionnalisation des principes de développement durable.

Mots-clés : banque, responsabilité sociale des entreprises, développement durable, théorie institutionnelle

\begin{abstract}
The integration of sustainable development in the banking corporate financing by Vincent Maymo and Valérie Pallas Saltiel

This paper focuses on the strategies aiming at integrating sustainable development (more particularly in its environmental dimension) into banks' practices regarding corporate financing. Beyond a motivation study, this paper brings together environment management literature and institutional theories. It provides an analytical scale to identify the institutionalisation processes of the overarching principles guiding sustainable development.

Keywords : bank, corporate social responsibility, sustainable development, institutional theory

\section{Resumen}

La integración del desarrollo sostenible en la financiación bancaria a las empresas por Vincent Maymo y Valérie Pallas Saltiel

Este artículo examina las estrategias para la integración del desarrollo sostenible (incluyendo la dimensión ambiental) en las prácticas bancarias de financiación de las empresas. Más allá de una discusión de las motivaciones, este documento recoge un entorno de gestión de la literatura y las teorías institucionales. Este articulo sugirió, un proceso de lectura de los procesos de institucionalización de los principios del desarrollo sostenible.
\end{abstract}

Palabras claves : banco, RSE, desarrollo sostenible, teoria institucional 


\section{L'intégration du développement durable dans le financement bancaire aux entreprises}

Alors que le secteur financier est a priori moins concerné que le secteur industriel par les problématiques de développement durable ${ }^{1}$, de nombreux auteurs soulignent l'impact des banques sur la société (B. Snoy, 1989 ; P. Thompson, C. Cowton, 2004 ; E. Marsiglia, I. Falautano, 2005 ; B. Scholtens, 2006, 2009 ; J. Andrew, 2007). L'activité de financement de ces dernières engage leur responsabilité financière et morale sur les projets de leurs clients (B. Scholtens, 2006, 2009). L'« intégration des préoccupations sociales et écologiques (...) à leurs activités commerciales et leurs relations avec leurs parties prenantes » (CE, 2001, p.8) relève d'une problématique de responsabilité sociale de l'entreprise ${ }^{2}$.

Les initiatives RSE des banques sont nombreuses, mais restent souvent réduites, aux dires des ONG (Les Amis de la terre, Bank track), aux impacts directs de leur activité : le respect des salariés, la réduction de la consommation d'eau ou du rejet de $\mathrm{CO} 2$, l'économie de papier. Ces actions, en marge de l'activité principale de l'entreprise et formalisées dans les documents de référence, relèvent de la communication ou du «cosmétique ». Pour être stratégiques et opérationnelles, les conséquences sociales et environnementales des activités des entreprises doivent être intégrées au cœur même de leur chaîne de valeur, en cohérence avec leur secteur d'activité (M. Porter, M. Kramer, 2006). Les impacts environnementaux des banques peuvent s'analyser, soit en interne au travers des processus organisationnels - «operating ecology»-, soit en externe au travers des produits - «product ecology» (M. Jeucken, J. Bouma, 1999 ; K. Tarna, 1999). Si les processus bancaires n'ont qu'un faible impact direct sur l'environnement ou la société en comparaison d'entreprises industrielles, il en va tout autrement des produits vendus par les banques qui accompagnent des entreprises clientes dont les externalités négatives peuvent être importantes (M. Jeucken, J. Bouma, 1999). Les banques sont ainsi engagées dans une chaine de responsabilité financière (F. Harvey, 2005).

Notre recherche défend l'idée que l'engagement responsable des banques se trouve dans leur cœur de métier et, en tant qu'intermédiaire financier, dans leurs activités de collecte et de financement. Le financement bancaire responsable auprès des PME reste un thème peu

\footnotetext{
${ }^{1}$ DD par la suite

${ }^{2}$ RSE par la suite
} 
exploré, contrairement à l'investissement socialement responsable ou au financement de projets internationaux de grande ampleur. Pourtant des initiatives se sont développées dans les banques depuis une quinzaine d'années, comme Prévair, un prêt à taux bonifié de la banque populaire d'Alsace soumis à la décision d'un comité de sélection (A. Berger, 2004). Par ailleurs, les banques représentent pour les PME des apports de ressources clés pouvant influencer leur stratégie d'intégration des objectifs de RSE (F. Quairel, M-N Auberger, 2005). La littérature souligne aussi l'intérêt de s'interroger sur le financement responsable de projets d'investissement de petite taille qui justifie un traitement différencié de ceux internationaux (B. Esty, 2004), les relations avec les PME offrant des perspectives importantes de développement des activités bancaires responsables (Delphi international, 1997).

Dans cet article, nous étudions les modalités d'intégration des principes de DD dans les pratiques de financement des banques auprès de leurs entreprises clientes. Outre les travaux sur la RSE, nous appuyons notre analyse sur la littérature en management environnemental et sur des entretiens auprès de responsables DD de banques françaises. L'enjeu de cette recherche est théorique et méthodologique. Théorique, par le croisement de la littérature sur le DD avec les théories institutionnelles. Méthodologique, au travers de la construction d'une grille de lecture visant à analyser le degré d'intégration des stratégies environnementales des banques françaises dans leur pratique de financement. Après avoir analysé les moteurs de l'intégration de la RSE dans le financement bancaire (1), nous étudions son processus d'institutionnalisation (2).

\section{L'enjeu d'integration de la RSE dans le financement bancaire.}

P. Bansal et K. Roth (2000) ont identifié trois principales motivations des entreprises industrielles à mener des initiatives écologiques : une recherche de compétitivité à long terme via du marketing vert; un souci de légitimation par une conformité réglementaire et une participation à des réseaux environnementaux ; un souci de responsabilité écologique à travers des donations. Dans le secteur bancaire, la prise en compte de la RSE conduirait à l'émergence d'un nouveau mode de fonctionnement des processus de financement des entreprises clientes. Cette évolution est autant à rechercher dans les motivations que dans les modalités de mise en œuvre. 


\subsection{Des motivations bancaires responsables.}

Les banques ont à la fois une légitimité structurelle à intégrer les principes de DD dans leur activité de financement et une raison concurrentielle de différenciation stratégique.

\subsubsection{Une légitimité structurelle établie.}

Dans la théorie de l'intermédiation financière, les banques justifient leur existence et leur position par leur supériorité informationnelle pour sélectionner et contrôler au mieux les emprunteurs et optimiser ainsi la gestion des ressources obtenues des déposants (D. Diamond, 1984 ; R. Ramakrishnan, A. Thakor, 1984). En effet, les banques réalisent des économies d'échelle, de gamme, de coût de production jointe, et de coûts de transaction par leur capacité à faire face aux imperfections du marché (G. Benston et al., 1976). Elles produisent également des activités complémentaires à leur activité d'intermédiation et bénéficient ainsi de synergies informationnelles (T. Campbell, W. Kracaw, 1980). Ce savoir supérieur en ferait des intermédiaires privilégiés pour mesurer les risques des entreprises en associant aux informations financières, comptables et économiques, des informations environnementales et sociales. Elles évalueraient et influenceraient ainsi le comportement socialement responsable de leurs entreprises clientes (B. Scholtens, 2006). Cette position est renforcée par les critiques adressées aux agences de notation extra-financières : une forte hétérogénéité des critères utilisés, un problème de fiabilité des données des entreprises évaluées (M. Porter, M. Kramer, 2006), une approche assez générale (B. Scholtens, 2009), une non prise en compte des caractéristiques économiques et sociales des entreprises évaluées (J. Igalens et al. 2007). La signature des principes équateurs par certaines banques s'inscrit dans cette logique d'arbitrage : sélectionner les projets en fonction des risques environnementaux et sociaux inhérents, les projets les plus risqués devant s'accompagner d'un plan responsable (C. Wright, A. Rwabizambuga, 2006 ; C. Wright, 2007 ; B. Scholtens, L. Dam, 2007).

Pour G. Palazzo et L. Rethel (2007), l'intermédiaire financier a un rôle de sauvegarde des actifs des déposants avec des implications sociales plus larges. De par sa fonction fiduciaire, il aurait des obligations éthiques professionnelles au même titre que les comptables, juristes ou médecins. Il y aurait ainsi un contrat implicite entre la banque et la société, la société reconnaissant l'existence de cette entité à condition qu'elle serve ses intérêts. 


\subsubsection{Une recherche de positionnement stratégique.}

Les motivations stratégiques des banques à intégrer les principes de DD dans leurs activités de financement sont abordées, soit en terme de meilleure gestion des risques, soit en terme d'opportunité de produits et de marchés. P. Thompson et C. Cowton (2004) montrent que les banques sont davantage préoccupées par la gestion des risques environnementaux en termes financiers que par l'exploitation d'opportunités de prêts.

Qu'ils soient directs (comme la pollution d'un terrain agricole donné en garantie d'un prêt), ou indirects (comme l'incapacité de l'entreprise cliente à s'adapter à de nouvelles réglementations environnementales ou consuméristes, entraînant un défaut de paiement), ces risques nécessitent pour les banques d'être gérés et minimisés (O. Weber et al., 2008a, 2008b). Cette préoccupation est devenue un impératif dès les années 80 , lorsque les banques américaines pouvaient être rendues responsables directement de la pollution environnementale de leurs clients et être obligées de payer des coûts d'assainissement (M. Jeucken, J. Bouma, 1999). Dans cette perspective, le DD est perçu comme une source de menaces contre lesquelles les banques doivent s'assurer en intégrant ces considérations dans leur processus d'octroi de crédit (P. Thompson, C. Cowton, 2004 ; O. Weber et al., 2008a, 2008b).

Les banques doivent aussi faire face à un risque de réputation lorsqu'elles financent des projets non responsables. C. Wright et A. Rwabizambuga (2006) montrent que l'adoption volontaire de codes de conduite répond à quatre types de stratégies de réputation :

- assurer les parties prenantes qu'on agit de manière responsable,

- se différencier des entreprises adoptant de mauvaises pratiques,

- adopter une stratégie globale de gestion des risques directs

- et enfin anticiper les pratiques irresponsables du secteur pouvant attirer l'attention des autorités réglementaires et de la société civile.

L'absence d'un véritable contrôle de la mise en œuvre des principes génère alors des échecs (M. Painter-Morland, 2006) : «green washing » (les banques adoptent des codes pour simuler un comportement exemplaire), «first world bias» (les codes ignorent les spécificités de l'entreprise et de son secteur), « oversight » (les conditions pour que les codes fonctionnent ne sont pas réunies). 
À l'opposé de ces logiques, la prise en compte de l'environnement initie des perspectives d'innovations de produits/services ou marchés bancaires. Des opportunités sont ouvertes pour financer, par exemple, des entreprises investissant dans des technologies respectueuses de l'environnement (P. Thompson, C. Cowton, 2004). Les produits et services verts bancaires s'accompagnent alors souvent de financements à des taux d'intérêts privilégiés (K. Tarna, 1999). Ce marché reste une niche stratégique (P. Thompson, C. Cowton, 2004), la réputation des banques n'étant pas renforcée par leur isomorphisme stratégique (D. Deephouse, S. Carter, 2005). Pourtant, même si BNP-Paribas a refusé pendant 5 ans de signer les principes Equateur, les banques agissant ainsi peuvent être confrontées à un risque de «back firing » sous la pression de la société civile (B. Scholtens, L. Dam, 2007).

Malgré les enjeux de gestion des risques et de positionnement stratégique, les initiatives RSE restent souvent isolées des unités opérationnelles ou fragmentées (M. Porter, M. Kramer, 2006). Des efforts ont été réalisés par certaines banques pour introduire des systèmes de management environnementaux visant à réduire les impacts directs et indirects de leur activité (Delphi International, 1997). Toutefois, ils se bornent essentiellement aux «activités de banque de gros » et les études réalisées ne soulignent pas de différence de pratique significative entre les signataires et les non signataires des chartes et principes éthiques (P. Thompson, C. Cowton, 2004).

En France, les pratiques des banques de détail font apparaître des préoccupations environnementales diverses qui n'exercent pas toujours d'impact direct sur la chaîne de valeur :

- des communications dans les rapports annuels sur les émissions de gaz à effet de serre ou encore la consommation d'énergies non renouvelables ;

- des discours sur les initiatives de DD intégrés dans les documents de référence (mécénat, présence dans les indices extra-financiers);

- des explorations en parallèle du cœur de métier (partenariats avec des institutions de l'économie solidaire, comme le Grameen Crédit Agricole) ;

- une sensibilisation du cœur de métier par des services joints : formation des commerciaux aux questions environnementales à la Société Générale, analystes du risque écologique au Crédit Suisse, experts techniques à la Banque Populaire ;

- la création de produits de financement DD intégrés : crédits «Prévair » de la Banque Populaire d'Alsace ; prêts HQE de Dexia. 
La plupart des banques s'engageraient donc dans des activités parallèles à leur activité principale, ne faisant pas une intégration complète des principes, cette dernière restant l'apanage des institutions financières de l'économie solidaire (F. Bensebaa, A. Béji-Bécheur, 2007).

\subsection{Les modalités d'intégration du développement durable.}

Les enjeux partenariaux occupent une position centrale dans les choix d'intégration des principes de DD. La nature et le poids des parties prenantes détermineraient le degré et les niveaux d'intégration de ces principes. L'opérationnalisation de telles stratégies invite à une réflexion sur la cohérence et la légitimité des outils de pilotage.

\subsubsection{Rôle des parties prenantes.}

La RSE est un auto-contrôle qui rend compte des obligations de l'entreprise à l'égard des groupes présents dans la société, autres que simplement ceux prescrits par la loi (T. Jones, 1980). M. Jeucken et J. Bouma (1999) catégorisent les stakeholders internes (actionnaires, conseil d'administration, employés) et externes (clients, fournisseurs, concurrents, média, ONG, autres institutions financières, société, gouvernements) des banques. E. Marsiglia et I. Falautano (2005), dans le domaine de la bancassurance, complètent cette liste par les agences de notation, auditeurs et membres de la supply chain, les institutions publiques garantes de la stabilité du système et de sa régulation, les associations professionnelles et les entreprises locales. I. Velaz et al. (2006) invitent les caisses d'épargne à tenir compte aussi des ONG, des centres de recherche et des institutions de formation.

Le comportement responsable tient compte des intérêts et enjeux des parties prenantes dans les décisions stratégiques, la gestion de leurs attentes s'opérant de façon, soit conflictuelle, soit consensuelle (M. Jensen, 2001). Les banques doivent alors fournir une variété d'informations et de réponses conséquentes, en utilisant des supports adéquats pour cibler différentes audiences : rapports annuels, rapports de développement durable et/ou sites web. Sur le plan environnemental, tous secteurs confondus, plusieurs types de parties prenantes peuvent exercer une pression sur les entreprises (Tableau 1). 
Tableau 1. Catégories de parties prenantes exerçant une pression environnementale.

\begin{tabular}{|l|l|l|}
\hline \multirow{2}{*}{$\begin{array}{c}\text { Catégories des parties } \\
\text { prenantes }\end{array}$} & \multicolumn{3}{|c|}{ Entités concernées } \\
\cline { 2 - 4 } & $\begin{array}{l}\text { I. Henriques, P. Sadorsky } \\
(1999)\end{array}$ & $\begin{array}{l}\text { S. Marquet-Pondeville et al } \\
(2007)\end{array}$ \\
\hline Réglementaires & $\begin{array}{l}\text { gouvernements, associations } \\
\text { professionnelles, réseaux } \\
\text { informels, concurrents }\end{array}$ & $\begin{array}{l}\text { législateurs régionaux, } \\
\text { nationaux, européens. }\end{array}$ \\
\hline Organisationnelles & $\begin{array}{l}\text { clients, fournisseurs, salariés, } \\
\text { actionnaires }\end{array}$ & $\begin{array}{l}\text { dirigeants, propriétaires } \\
\text { /actionnaires, salariés }\end{array}$ \\
\hline Communautaires & $\begin{array}{l}\text { groupes communautaires, } \\
\text { organisations } \\
\text { environnementales, autres } \\
\text { lobbies potentiels }\end{array}$ & $\begin{array}{l}\text { communautés } \\
\text { presse/media, } \\
\text { environnementales, instituts } \\
\text { scientifiques }\end{array}$ \\
\hline Média / Marché & \multicolumn{2}{|c|}{$\begin{array}{l}\text { acheteurs, concurrents, } \\
\text { associations professionnelles }\end{array}$} \\
\hline
\end{tabular}

Certaines entreprises intègrent l'ensemble des parties prenantes dans l'élaboration de leur stratégie environnementale alors que d'autres ne s'intéressent qu'à certaines, considérées comme légitimes. Les parties prenantes organisationnelles, communautaires et de marché auraient une influence positive et significative sur la stratégie environnementale (S. MarquetPondeville et al., 2007). En outre, le degré d'intégration serait contingent à la réglementation du secteur d'activité (S. Marquet-Pondeville et al., 2007 ; S. Sharma, 2000).

A l'instar de C. Hunt et E. Auster (1990) et N. Roome (1992), I. Henriques et P. Sadorsky (1999) ont adapté le modèle de A. Carroll (1979) sur les niveaux d'intégration de la RSE dans la stratégie d'entreprise à la seule dimension environnementale. Quatre niveaux sont retenus :

- stratégie réactive (nier toute responsabilité),

- $\quad$ stratégie défensive (admettre la responsabilité mais se défendre),

- stratégie accommodante (accepter la responsabilité et faire ce qui est nécessaire),

- stratégie proactive (anticiper la responsabilité et faire ce qui est requis).

Plusieurs profils d'entreprise en découlent (Tableau 2). Les agences de notation sociale proposent, elles aussi, leur propre typologie des degrés d'engagement des entreprises en matière de RSE (voir E. Alberola et N. Richez-Battesti (2005) pour celle de Vigeo). 
Tableau 2. Stratégies environnementales et perception des parties prenantes (1: faible, 4: fort)

\begin{tabular}{|l|l|l|l|l|}
\hline Parties prenantes/Stratégies & Réactive & Défensive & Accommodante & Proactive \\
\hline Réglementaires & $\mathbf{1}$ & $\mathbf{3}$ & $\mathbf{4}$ & $\mathbf{2}$ \\
\hline Communautaires & $\mathbf{2}$ & $\mathbf{1}$ & $\mathbf{3}$ & $\mathbf{4}$ \\
\hline Organisationnelles & $\mathbf{1}$ & $\mathbf{2}$ & $\mathbf{3}$ & $\mathbf{4}$ \\
\hline Media & $\mathbf{4}$ & $\mathbf{2}$ & $\mathbf{3}$ & $\mathbf{1}$ \\
\hline
\end{tabular}

Source : élaboré à partir des résultats de I. Henriques et P. Sadorsky (1999)

D'un côté, les entreprises proactives sont dotées d'un plan environnemental formalisé et d'une entité spécialement dédiée à la sécurité environnementale. Elles accordent de la valeur à l'ensemble des parties prenantes (sauf les media). D'un autre côté, les entreprises réactives sont davantage préoccupées par leur image médiatique que par une sanction possible du régulateur. Elles n'accordent pas d'importance aux parties prenantes à l'exception des médias. Il semblerait que les entreprises bancaires justifient leur orientation partenariale par la légitimité de la partie prenante ou par le cadre institutionnel. Elles accorderaient ainsi plus d'importance aux parties prenantes réglementaires qu'à celles organisationnelles. C'est le cas des banques britanniques qui, ne souhaitant pas être impliquées directement dans la collecte d'information environnementale, se contentent d'une information déclarative présente dans les rapports annuels en respect des réglementations et normes environnementales (P. Thompson, C. Cowton, 2004).

Pour autant, les banques n'échappent pas aux pressions des autres parties prenantes : l'affaire de l'usine de papier en Uruguay dénoncée par Bank Track est devenue un conflit politique tel que Calyon, financeur du projet, a été amené à généraliser les principes Equateur. Cette réaction est cohérente avec la littérature qui montre que les media exercent une influence sur les questions environnementales davantage en cas de crise que de réussite environnementale (I. Henriques, P. Sadorsky, 1999). En France, parmi les banques interrogées, deux positionnements émergent :

- attendre que les parties prenantes, considérées comme légitimes (actionnaires ou sociétaires), s'expriment sur la RSE pour s'engager dans la démarche.

- attendre une évolution du cadre institutionnel pour créer de nouveaux produits. 
Ces stratégies d'intégration du DD nécessitent une adaptation des systèmes de contrôle et de pilotage dans un souci de cohérence et de légitimité.

\subsubsection{Cohérence et légitimité des outils de pilotage.}

Selon K. Basu et G. Palazzo (2008), la politique de RSE se développe au travers d'un processus à trois niveaux :

- les principes qui gouvernent la relation de l'entreprise avec ses parties prenantes ;

- les discours par lesquels l'organisation justifie son engagement ;

- les pratiques organisationnelles.

La performance sociale et environnementale est alors une configuration organisationnelle de principes, de politiques et de résultats observables (D. Wood, 1991). Le pilotage de l'intégration de la politique environnementale s'analyse donc autant dans les principes et les discours que dans les comportements et leurs régularités. Il questionne d'une part, l'influence du type de stratégie environnementale de l'entreprise sur les caractéristiques de ses systèmes de pilotage et de contrôle et d'autre part, les outils de pilotage mobilisés pour mesurer la performance environnementale et déployer la stratégie environnementale à tous les niveaux de l'organisation.

Concernant la première préoccupation, S. Marquet-Pondeville et al. (2007) ont établi une relation directe entre la stratégie environnementale d'une entreprise et les systèmes de contrôle de gestion environnementaux mis en œuvre : plus les entreprises sont proactives, plus elles mettent en place un ensemble varié de systèmes de contrôle de gestion environnementaux.

Concernant la deuxième préoccupation, selon qu'il s'agisse d'un contrôle a priori, visant à piloter la diffusion de la stratégie au cœur de l'entreprise, ou a posteriori, visant à contrôler les résultats environnementaux de l'entreprise, les outils mobilisés diffèrent dans leur conception. A. Dohou et N. Berland (2007) identifient la comptabilité environnementale, les normes ISO $14001^{3}$ et la norme EMAS ( «Eco Management and Audit Scheme ») comme des outils de pilotage pour la seule dimension environnementale de la RSE. Parmi les outils de pilotage de la stratégie, le balanced scorecard, reconnu comme un vecteur de communication de la

\footnotetext{
${ }^{3}$ ISO 14001 fournit un canevas d'accompagnement à la mise en place d'un système de management environnemental en spécifiant les structures de responsabilité, les procédures et les outils de pilotage et de reporting.
} 
stratégie auprès des entités opérationnelles (R. Kaplan, D. Norton, 1998), serait pertinent pour déployer une stratégie de DD et mesurer sa performance (M. Epstein, P. Wisner, 2001). D'autres outils de mesure de la performance globale du DD existent : le triple bottom line reporting et le reporting du GRI. La production volontaire de ces outils reposerait sur un contrat social implicite avec les parties prenantes (F. Hui, G. Bowrey, 2008).

Pour autant, l'existence de tels outils n'est pas garante de la performance responsable de l'entreprise. On pourrait assister à un phénomène de découplage (J. Meyer et B. Rowan, 1977) entre les structures intégrant les principes de DD et les activités. Les organisations isomorphes maintiennent leur légitimité en institutionnalisant fortement dans leur structure formelle des pratiques et procédures légitimées en externe plutôt qu'en termes d'efficience et fonctionnant comme des mythes. Les règles cohérentes avec les activités de l'organisation peuvent alors entrer en conflit avec les règles externes. Dès lors, toute tentative de contrôle et de coordination au service de l'intégration du DD peut conduire à une perte de légitimité et à un découplage. Ce management symbolique (B. Ashforth, B. Gibbs, 1990) consiste à agir de telle sorte que l'entreprise apparaisse conforme aux attentes et aux valeurs sociales. Dans ce cas, l'entreprise n'a pas à modifier son système de production et n'a pas réellement à satisfaire les attentes des acteurs tant qu'elle apparaît légitime. La diffusion d'information sociétale sous forme de reporting peut s'inscrire dans cette démarche. En matière de DD, un tel découplage pourrait avoir pour cause des conflits entre les demandes du public et de la réglementation et les intérêts financiers des managers. Le découplage serait donc plus probable dans les organisations qui orientent l'éthique exclusivement vers la communication (G. Weaver et al. 1999). Pour isoler ces effets de découplage et maîtriser les risques partenariaux, il est alors tentant, avec J. Weber (1981, 1993), de défendre l'idée d'une institutionnalisation des principes de DD consistant à «intégrer l'éthique dans la prise de décision quotidienne et les pratiques de travail des salariés » (J. Weber, 1981, p.47).

\section{2. le processus d'Institutionnalisation du DD dans les pratiques bancaires.}

Les théories institutionnelles permettent d'étudier le processus d'institutionnalisation des principes de DD dans les pratiques des organisations. Une grille d'analyse de l'intégration de ces principes dans les décisions de financement aux entreprises clientes est élaborée. Ses modalités d'opérationnalisation sont enfin étudiées. 


\subsection{L'apport des théories institutionnelles.}

Les institutions «sont constituées de structures et d'activités cognitives, normatives et de régulation qui fournissent de la stabilité et du sens au comportement social. Elles sont transportées par des supports variés (cultures, structures et routines) et opèrent à des niveaux multiples de compétences » (R. Scott, 1995, p.33). Trois piliers identifient les éléments constitutifs des institutions (tableau 3).

Tableau 3. Les trois piliers des institutions

\begin{tabular}{|c|c|c|c|}
\hline & Régulation & Normatif & Cognitif \\
\hline $\begin{array}{l}\text { Courants de } \\
\text { pensée }\end{array}$ & $\begin{array}{c}\text { Néo- } \\
\text { institutionnalistes : } \\
\text { économistes (North) }\end{array}$ & $\begin{array}{c}\text { Sociologues } \\
\text { (Durkheim, Parsons, } \\
\text { Selznick) }\end{array}$ & $\begin{array}{c}\text { Sociologues du nouvel } \\
\text { institutionnalisme } \\
\text { (Berger, Meyer, Powell, } \\
\text { DiMaggio, Zucker) }\end{array}$ \\
\hline $\begin{array}{l}\text { Hypothèse des } \\
\text { acteurs }\end{array}$ & Opportunisme & Obligation sociale & $\begin{array}{c}\text { Construction sociale des } \\
\text { acteurs }\end{array}$ \\
\hline $\begin{array}{l}\text { Mécanismes de } \\
\text { contrôle }\end{array}$ & Coercition & Normatif & Mimétique \\
\hline Logique & Instrumentalisme & Justesse & Orthodoxie \\
\hline Indicateurs & $\begin{array}{l}\text { Règles, lois, } \\
\text { sanctions }\end{array}$ & $\begin{array}{l}\text { Certification, accréditation } \\
\text { (valeurs, normes) }\end{array}$ & $\begin{array}{l}\text { Isomorphisme, } \\
\text { prédominance. }\end{array}$ \\
\hline $\begin{array}{ll}\text { Bases } & \text { de } \\
\text { légitimité } & \end{array}$ & $\begin{array}{l}\text { Légalement } \\
\text { sanctionné }\end{array}$ & Moralement gouverné & Culturellement appuyé \\
\hline $\begin{array}{l}\text { Logiques qui } \\
\text { déterminent } \\
\text { l'action sociale }\end{array}$ & $\begin{array}{l}\text { Les intérêts des } \\
\text { individus sont } \\
\text { donnés. }\end{array}$ & $\begin{array}{l}\text { Le choix est fondé sur un } \\
\text { contexte social et orienté par } \\
\text { une dimension morale qui } \\
\text { prend en compte les relations } \\
\text { et obligations des autres. }\end{array}$ & $\begin{array}{l}\text { Les croyances culturelles } \\
\text { et les conceptions propres } \\
\text { des acteurs sont le produit } \\
\text { des processus sociaux. }\end{array}$ \\
\hline
\end{tabular}

Source : élaboré à partir de R. Scott (1995)

Dans l'approche néo-institutionnaliste, premier pilier, l'environnement, conçu comme cadre institutionnel, structure les comportements des acteurs et justifie les pratiques organisationnelles (P. DiMaggio, W. Powell, 1983). Les institutions contraignent et régularisent le comportement des acteurs. Le secteur bancaire est particulièrement concerné par de fortes pressions institutionnelles au niveau international (standards de Bâle) ou national (loi NRE) : les régulateurs sont des parties prenantes conférant aux banques une légitimité régulatrice (D. Deephouse, 1996 ; D. Deephouse, S. Carter, 2005). 
Dans le second pilier, les règles normatives introduisent une dimension prescriptive, évaluative et obligatoire à la vie sociale. Les acteurs sont encastrés dans un réseau complexe de règles et de croyances conventionnelles. Les principes Equateur soutenus par $85 \%$ du marché du financement de projets dans les pays développés (B. Scholtens, L. Dam, 2007) illustrent ce principe de soft law.

Enfin, dans une approche cognitive des institutions, les individus construisent et négocient la réalité sociale, dans un contexte culturel préexistant. Ainsi de nombreuses banques ont repris à leur compte le crédit «PROVair » initié par la Banque Populaire d'Alsace.

Deux dynamiques d'institutionnalisation apparaissent : l'une descendante lorsque le cadre institutionnel influence les comportements; l'autre émergente par laquelle les acteurs dessinent les institutions. Trois phénomènes moteurs de cette dynamique et qualifiés par P. DiMaggio et W. Powell (1991) d'isomorphismes, sont alors identifiés : coercitif dans le modèle de régulation, normatif dans le modèle normatif et mimétique dans le modèle cognitif. - L'isomorphisme coercitif d'une organisation est déterminé par les pressions formelles et informelles d'autres organisations dont elle est dépendante et par des attentes culturelles.

- L’isomorphisme normatif est déterminé par la professionnalisation (diplôme, participation des managers à des associations professionnelles), elle-même influencée par les pressions coercitives et d'imitation.

- L’isomorphisme mimétique dépend de l'incertitude de la relation entre la fin et les moyens, et de l'ambiguïté des buts poursuivis par l'organisation. Les organisations subissant cette incertitude tentent de prendre alors exemple sur des organisations similaires de leur champ qu'elles perçoivent comme étant plus légitimes ou qui ont plus de succès.

\subsection{La construction d'une grille de lecture.}

Les développements précédents ont identifié plusieurs niveaux d'intégration de la stratégie de DD des banques pouvant correspondre à différents niveaux d'institutionnalisation :

- d'un côté, le travail de I. Henriques et P. Sadorsky (1999) met en exergue quatre catégories de stratégie environnementale ;

- d'un autre côté, l'approche institutionnaliste (P. DiMaggio, W. Powell, 1983; R. Scott, 1995) distingue trois isomorphismes institutionnels.

Le croisement de ces deux approches conduit à la grille de lecture suivante (tableau 4). 
Tableau 4. Grille de lecture des processus d'institutionnalisation des stratégies de DD

\begin{tabular}{|l|l|l|l|}
\hline \multicolumn{1}{|c|}{ Isomorphisme institutionnel } & Coercitif & Normatif & Mimétique \\
Stratégies environnementales & & & \\
\hline Réactives & & & \\
\hline Défensives & & & \\
\hline Accommodantes & & & \\
\hline Proactives & & & \\
\hline
\end{tabular}

L'enjeu de cette grille de lecture est d'ordre méthodologique. La banque devient le lieu d'institutionnalisation de pratiques où les règles descendantes constituent un cadre institutionnel aux côtés de pratiques autonomes. D'une approche interorganisationnelle qui met la banque en relation avec son cadre institutionnel, nous déplaçons l'analyse vers une dimension intraorganisationnelle, celle des activités et des processus. Les trois isomorphismes sont étudiés comme des leviers de l'institutionnalisation des stratégies de DD bancaires :

- coercitive, en identifiant les outils de gestion qui structurent les comportements des acteurs bancaires dans leurs schémas de travail quotidiens.

- normative, en relevant les normes professionnelles et de travail ;

- mimétique, en appréhendant les processus d'apprentissage et de créativité auxquels se livrent les acteurs bancaires dans leurs pratiques quotidiennes.

Chaque banque peut ainsi être caractérisée à un moment donné par un modèle différent d'institutionnalisation des stratégies de DD dans leurs activités de financement d'entreprise. Par exemple,

- Certaines auraient un pilier coercitif fortement marqué, voire des normes très présentes et pour autant, mobiliseraient peu de mécanismes d'apprentissage et d'innovation, tendant à se comporter plutôt comme des banques réactives, voire défensives dans leur stratégie environnementale.

- D'autres, plus proactives, intègreraient plus fortement la dimension mimétique, ne se contentant pas d'une institutionnalisation du DD limitée à de la communication auprès de 
leurs parties prenantes. Ces banques proactives auraient atteint le niveau le plus élevé d'institutionnalisation des stratégies de DD en intégrant les trois piliers.

Cet ancrage méthodologique suppose de ne pas concevoir les modèles d'institutionnalisation de façon statique, mais dynamique, en étudiant pour une banque donnée, le passage d'un modèle à un autre. Les banques pourraient ainsi évoluer d'un modèle défensif vers un modèle proactif ou inversement. En ce sens, le tableau 4 s'étudie comme un damier où les banques définissent en continu leurs domaines d'intégration du DD.

\subsection{Les modalités d'opérationnalisation de la grille de lecture.}

F. Bensebaa et A. Béji-Bécheur (2007) proposent des pistes pour la confrontation au terrain : une analyse des discours, des techniques de contrôle et des pratiques organisationnelles associées. Les comportements des acteurs doivent être analysés dans les pratiques effectives qu'ils mettent en œuvre au quotidien (A. Argandona, 2007). Il s'agit pour nous de comprendre comment les décisions quotidiennes envers les entreprises clientes intègrent la dimension DD, à partir de l'analyse des trois dimensions du processus d'institutionnalisation: coercitive, normative et mimétique.

\subsubsection{L'intégration coercitive de la stratégie de DD.}

Dans ce prisme, les acteurs bancaires sont conduits par des intérêts propres et acceptent l'existence de règles sans reconnaître ou croire en leur justice. L'institutionnalisation coercitive de la stratégie de DD structure, oriente et suit, à l'aide d'outils et de procédures, les comportements des acteurs bancaires envers leurs entreprises clientes.

Ces mécanismes organisationnels élaborés par les fonctions support (contrôle de gestion, ressources humaines, marketing) reflètent une légitimité organisationnelle managériale (M. Ruef, R. Scott, 1998). La création de produits en lien avec la réglementation, la mise en place d'outils de pilotage et de mesure de la performance environnementale, la réalisation d'audits du système du DD ainsi que les processus d'évaluation et d'incitation du personnel à la performance environnementale concourent à une intégration coercitive de la stratégie de DD. R. Sroufe et al. (2002) définissent ces systèmes de management environnemental comme des systèmes formels qui intègrent des procédures de formation, de pilotage et de contrôle de la performance environnementale. Des exemples de systèmes de management responsable existent dans d'autres secteurs (F. Perriniand, M. Minoja, 2008). Par ailleurs, les banques de 
soutien aux pays en développement sembleraient concernées par une institutionnalisation coercitive de la stratégie de DD, leur processus de prise de décision suivant une logique technocratique qui vise à produire des résultats mesurables, rapidement et efficacement (C. Wright, 2007).

Proposition 1 : Les banques commerciales qui privilégieraient ce pilier coercitif par rapport aux deux autres verraient leurs procédures et outils découplés des activités réellement menées par les acteurs. La plupart des procédures et outils seraient présents dans les rapports annuels ou rapports de DD uniquement pour communiquer l'engagement des dirigeants dans une stratégie de DD. Les banques concernées adopteraient ainsi des stratégies réactives (et dans une moindre mesure défensives) en matière de $\mathrm{DD}$, consistant à nier toute responsabilité environnementale ou à se défendre de ne pas intégrer ces principes dans les comportements quotidiens.

\subsubsection{L'intégration normative de la stratégie de DD.}

Certains facteurs influencent les valeurs dans l'entreprise. Ils sont multidimensionnels et varient d'un individu et d'une organisation à l'autre, la cohérence entre les valeurs s'ajustant au final sur des motivations instrumentales et normatives (F. Perriniand, M. Minoja, 2008). L'institutionnalisation normative de la stratégie de DD consiste en une diffusion, descendante ou non, des normes et standards de travail définis par l'organisation et/ou la profession pour structurer et réguler les comportements et choix des acteurs bancaires engagés auprès des entreprises clientes. La sensibilisation, l'information et la formation du personnel concourent à une intégration normative de la stratégie de DD. Ces normes et standards professionnels, qui concernent essentiellement les critères de prise de décision et de suivi de la clientèle, reflètent une légitimité organisationnelle technique (M. Ruef, R. Scott, 1998). Il en est ainsi des normes d'évaluation et de dépistage ex ante des risques environnementaux ainsi que des normes d'évaluation ex post du respect par les entreprises clientes des contraintes environnementales. Il s'agit notamment, en référence aux travaux de O. Weber et al. (2008a, 2008b), d'identifier comment les banques intègrent dans leurs procédures et les différentes phases du processus de gestion du risque de crédit (notation, coût, tarification et contrôle), les risques environnementaux subis par les entreprises financées. Ces éléments relèvent des codes de conduite volontaires d'engagement (C. Wright, A. Rwabizambuga, 2006) auxquels de nombreuses banques ont adhéré comme les principes UNEP et les principes Equateur. 
Selon l'étude de P. Thompson et C. Cowton (2004), la majorité des banques interrogées adoptent une politique formelle de prêt d'entreprise en incorporant des considérations environnementales. B. Scholtens (2009) aboutit à la même conclusion. La différence entre les banques signataires et non signataires des principes UNEP ne porte pas sur leur pratique d'appréciation ou non des risques d'environnementaux mais sur les raisons qui les conduisent à y recourir : les signataires considèrent que la poursuite du DD et l'adhésion à une prise de position éthique sont des raisons plus importantes que les non signataires. Par ailleurs, les banques interrogées disent ne pas refuser de prêter à des entreprises appartenant à un secteur particulier mais attendent d'elles qu'elles répondent à des normes environnementales connues.

Proposition 2: Les banques qui associeraient au pilier coercitif ce pilier normatif montreraient qu'elles sont conscientes qu'il importe d'intégrer la stratégie de DD au cœur du métier bancaire (en particulier dans la prise de décision de financement en amont et en aval), se comportant ainsi plutôt comme des banques accommodantes. Dans ce cas, les normes DD ne seraient pas découplées des activités réelles. Mais d'un autre point de vue, en adoptant des comportements conformes à ces normes, les acteurs bancaires pourraient être amenés à les justifier auprès de leur clientèle, en cas de nécessité, par l'évidence, se comportant ainsi davantage comme des banques défensives. Les actions sont en effet justifiables en référence à des règles et des croyances qui ne sont plus remises en cause dès lors qu'elles sont institutionnalisées (P. DiMaggio, 1988 ; P. DiMaggio, W. Powell, 1991).

\subsubsection{L’intégration mimétique de la stratégie de DD.}

La dimension cognitive des processus isomorphes est l'imitation. D'un point de vue cognitif, les acteurs sont considérés comme actifs avec des intérêts, des droits et des capacités pour l'action, qui ne sont pas déterminés naturellement. Leurs comportements balancent entre autonomie et suivi de la norme : autonomes, ils sont construits socialement mais, en situation d'incertitude, ils peuvent se comporter de façon conventionnelle en utilisant des guides pour donner du sens à leurs actions. Les fondements cognitifs du DD peuvent alors s'observer dans les routines et les conventions qui étudient l'évolution des régularités comportementales individuelles et interpersonnelles (P-Y Gomez, B. Jones, 2000 ; N. Postel, S. Rousseau, 2008). «L'organisation va infuser un comportement socialement responsable non pas à travers des procédures mais des attitudes, non pas via des directives mais des exemples, non pas «à cause d'eux » mais à cause de «nous»». (H. Mintzberg 1983). La RSE pourrait résulter des valeurs et convictions personnelles de certains managers (F. Perriniand, 
M. Minoja, 2008) et d'initiatives individuelles proactives (C. Wright, A. Rwabizambuga, 2006). Des valeurs imposées à une organisation pourraient être en conflit direct avec les propriétés émergentes de cette organisation, conduisant ainsi à une rupture de l'intégrité organisationnelle (M. Painter-Morland, 2006). La question de l'apprentissage est donc au cœur de l'intégration du DD : chaque acteur apprend de nouveaux modes de fonctionnement cohérents avec les principes environnementaux qui lui permettent de prendre une décision compte tenu des informations dont il dispose. La phase d'institutionnalisation mimétique de la stratégie de DD consisterait alors pour les acteurs bancaires à intégrer les principes de DD audelà des normes prescrites par la banque.

Proposition 3 : Les banques qui associeraient au pilier coercitif et au pilier normatif le pilier mimétique montreraient une intégration totale de la stratégie de DD au cœur du métier bancaire, se comportant ainsi plutôt comme des banques proactives. Innovatrices dans les pratiques d'engagement auprès des entreprises en matière de $\mathrm{DD}$, elles pourraient ensuite amener d'autres banques à s'engager dans la même voie.

\section{Conclusion}

Les hésitations des banques à intégrer les principes de DD dans leur stratégie et dans sa mise en œuvre interrogent doublement : d'une part le rôle central des banques dans le financement de l'économie justifie leur avantage informationnel pour constituer des vecteurs privilégiés de diffusion du DD ; d'autre part, une approche stratégique de la question conduit à souligner l'intérêt d'une telle intégration. Notre contribution se veut à la fois méthodologique et théorique. Sur le plan méthodologique, nous nous sommes attachés à analyser les différentes dimensions de l'intégration du DD dans les stratégies bancaires. Sur le plan théorique, il s'agissait de structurer l'analyse du processus d'intégration.

Nous avons donc interprété les différents niveaux d'intégration des principes de DD comme un processus d'institutionnalisation d'une innovation managériale. Plusieurs approches théoriques pouvaient être mobilisées. Parmi celles-ci, l'approche institutionnaliste présente l'avantage d'apporter une lecture dynamique et multi niveaux des processus d'institutionnalisation. Elle permet notamment de définir plusieurs scénarii d'intégration du DD dans la stratégie des banques. Ainsi, les banques s'engageant dans un processus d'intégration de type réactif sont susceptibles d'encourir des risques de découplage. À l'opposé, les banques s'engageant pro activement dans l'intégration du DD sont susceptibles 
de bénéficier d'avantages en termes d'assurance, de maîtrise de la relation client et de légitimité.

\section{Bibliographie :}

Alberola E., Richez-Battesti N. De la responsabilité sociétale des entreprises : Évaluation du degré d'engagement et d'intégration stratégique. La Revue des Sciences de Gestion, Direction et Gestion, 2005, 211-212, 55-69.

Andrew J. The Equator Principles, Project Finance and the Challenge of Social and Environmental Responsibility. Issues in Social and Environmental Accounting, 2007, 1(1), 40-53.

Argandona A. Integrating Ethics into Action Theory and Organizational Theory. Journal of Business Ethics, 2007, 78(3), 435-446.

Ashforth B., Gibbs B. The Double Edge of Organizational Legitimation. Organization Science, 1990, 1(2), 177-195.

Bansal P., Roth K. Why Companies Go Green: a Model of Ecological Responsiveness. Academy of Management Journal, 2000, 43(4), 717-736.

Basu K., Palazzo G. Corporate Social Responsibility : a Process Model of Sensemaking. Academy of Management Review, 2008, 33(1), 122-136.

Bensebaa F., Béji-Bécheur A. Institutionnalisation et rationalisation des pratiques de RSE. Finance Contrôle Stratégie, 2007,10(2), 63-95.

Benston G., Smith Jr., Clifford W. Transaction Cost Approach to the Theory of Financial Intermediation. The Journal of Finance, 1976, 31(2), 215-231.

Berger A. Transparence bancaire dans le financement du développement durable. Entreprise Ethique, avril 2004, 20, 43-48.

Campbell T., Kracaw W. Information Production, Market Signalling and the Theory of Financial Intermediation. The Journal of Finance, 1980, 35(4), 863-882.

Carroll A. A Three-Dimensional Conceptual Model of Corporate Social Performance. Academy of Management Review, 1979, 4(4), 497-505.

Commission des Communautés Européennes. Livre vert, promouvoir un cadre européen pour la RSE. 2001, juillet, Bruxelles.

Delphi international. The Role of Financial Institutions in Achieving Sustainable Development. Report to the European commission, 1997, November, Executive Summary 7 p.

Deephouse D. Does isomorphism legitimate? Academy of Management Journal, 1996, 39(4), 10241039.

Deephouse D., Carter S. An Examination of Differences Between Organizational Legitimacy and Organizational Reputation. Journal of Management Studies, 2005, 42(2), 229-360.

Diamond D. Financial Intermediation and Delegated Monitoring. Review of Economic Studies, 1984, 51(166), 393-414.

DiMaggio P. Interest and Agency in Institutional Theory, in Zucker L. Institutional Patterns and Organizations: Culture and Environnement, 1988, Cambridge, Ballinger.

DiMaggio P., Powell W. The Iron Cage Revisited: Institutional Isomorphism and Collective rationality in Organizational Fields. American Sociological Review, 1983, 48(2), 147-160.

DiMaggio P., Powell W. Introduction, in Powell W., DiMaggio P. (Eds.), The New Institutionalism in Organizational Analysis, 1991, The Chicago University Press.

Dohou A., Berland N. Mesure de la performance globale des entreprises. Association francophone de comptabilité, 2007, Poitiers. 
Epstein M., Wisner P. Using a Balanced Scorecard to Implement Sustainability. Environmental Quality Management, 2001, 11(2), 1-10.

Esty B. Why Study Large Projects? An Introduction to Research on Project Finance. European Financial Management, 2004, 10(2), 213-224

Gomez P-Y, Jones B. Conventions: An Interpretation of Deep Structure in Organizations. Organization Science, 2000, 11(6), 696-708.

Harvey F. Rise of the Ethical Financier: The FT Sustainable Banking Awards. Financial Times, November 2005, p.13.

Henriques I., Sadorsky P. The Relationship between Environmental Commitment and Managerial Perceptions of Stakeholder Importance. Academy of Management Journal, 1999, 42(1), 87-99.

Hui F., Bowrey G. Corporate Social Responsibility Reporting of Two Note-issuing Banks in Hong Kong. Working Paper 2008/02, University of Wollongong.

Hunt C., Auster E. Proactive Environmental Management: Avoiding the Toxic Trap. Sloan Management Review, 1990, 31(2), 7-18.

Igalens J., Dejean F., El-Akremi A. Etude sur la notation sociétale: Influence des systèmes économiques et sociaux. Conférence Internationale de Management Stratégique, 6-9 Juin 2007, Montréal.

Jensen M. Value Maximisation, Stakeholder Theory, and the Corporate Objective Function. European Financial Management, 2001, 7(3), 297-317.

Jeucken M., Bouma J. The Changing Environment of Banks. Greener Management International, 1999, 27, 21-35.

Jones T. Corporate Social Responsibility Revisited, Redefined. California Management Review, 1980, 22(3), 206-221.

Kaplan R., Norton D. Le tableau de bord prospectif. Pilotage stratégique : les 4 axes du succès. Les Editions d'Organisation, 1998, Paris.

Marquet-Pondeville S., Swaen V., de Rongé Y. The Impact of External Environment, Stakeholders' Pressures and Environmental Strategy on Environmental Management Control Systems. Association Francophone de Comptabilité, 2007, Poitiers.

Marsiglia E., Falautano I. Corporate Social Responsibility and Sustainability Challenges for a Bancassurance Company. The Geneva Papers, 2005, 30, 485-497.

Meyer J., Rowan B. Institutionalized Organizations: Formal Structure as Myth and Ceremony. American Journal of Sociology, 1977, 83(2), 340-363.

Mintzberg H. The Case for Corporate Social Responsibility. Journal of Business Strategy, 1983, 4(2), $3-15$.

Painter-Morland M. Triple Bottom-line Reporting as Social Grammar:Integrating Corporate Social Responsibility and Corporate odes of onduct. Business Ethics: A European Review, 2006, 15(4), 352364.

Palazzo G., Rethel L. Conflicts of Interest in Financial Intermediation. Journal of Business Ethics, 2007, 81, 193-207.

Perriniand F., Minoja M.. Strategizing Corporate Social Responsibility:Evidence from an Italian Medium-Sized Family-Owned Company. Business Ethics: A European Review, 2008, 17(1), 47-63.

Porter M., Kramer M. Strategy and Society:The Link Between Competitive Advantage and Corporate Social Responsibility. Harvard Business Review, 2006, 84(12), 78-92.

Postel N., Rousseau S. RSE et éthique d'entreprise : la nécessité des institutions. M@n@gement, 2008, 11(2), 137-160.

Quairel F., Auberger M-N. Management responsable et PME : Une relecture du concept de "responsabilité sociétale de l'entreprise. La Revue des Sciences de Gestion, Direction et Gestion, 2005, 211-212, 111-126. 
Ramakrishnan R., Thakor A. Information Reliability and a Theory of Financial Intermediation. Review of Economic Studies, 1984, 51(166), 415-432.

Roome N. Developing Environmental Management Systems. Business Strategy \& the Environment, 1992, 1, 11-24.

Ruef M., Scott R. A Multidimensional Model of Organizational Legitimacy: Hospital Survival in Changing Institutional Environnnents. Administrative Science Quarterly, 1998, 43, 877-904.

Scholtens B. Finance as a Driver of Corporate Social Responsibility. Journal of Business Ethics, 2006, 68, 19-33.

Scholtens B. Corporate Social Responsibility in the International Banking Industry. Journal of Business Ethics, 2009, 86, 159-175.

Scholtens B., Dam L. Banking on the Equator. Are Banks that Adopted the Equator Principles Different from Non-Adopters? World Development, 2007, 35(8), 1307-1328.

Scott R. Contemporary Institutional Theory. in Institutions and Organizations. Sage, 1995.

Sharma S. Managerial Interpretations and Organizational Context as Predictors of Corporate Choice of Environmental Strategy. Academy of Management Journal, 2000, 43(4), 681-697.

Snoy B. Ethical Issues in International Lending. Journal of Business Ethics, 1989, 8(8), 635-639.

Sroufe R., Montabon F., Narasimhan R., Wang X. Environmental Management Practices: A Framework. Greener Management International, 2002, 40, 23-44.

Tarna K. Reporting on the Environment. Current Practice in the Financial Services Sector. Greener Management International, 1999, 27, 49-64.

Thompson P., Cowton C. Bringing the Environment into Bank Lending: Implications for Environmental Reporting. The British Accounting Review, 2004, 36, 197-218.

Velaz I., Sison A., Fontrodona J. Incorporating CSR and Stakeholder Management into Corporate Strategy: a Case Study of the CAN Expérience 2002-2006. Corporate Governance, 2006, 7(4), 434445.

Weaver G., Trevino L., Cochran P. Integrated and Decoupled Corporate Social Performance: Management Commitments, External Pressures, and Corporate Ethics Practices. Academy of Management Journal, 1999, 42(5), 539-552.

Weber J. Institutionalizing Ethics into the Corporation. MSU Business Topics, 1981, 29(2), 47-52.

Weber J. Institutionalizing Ethics into Business Organizations: a Model and Research Agenda. Business Ethics Quarterly, 1993, 3(4), 419-436.

Weber O., Fenchel M., Scholz R. Empirical Analysis of the Integration of Environmental Risks into the Credit Risk Management Process of European Banks. Business Strategy and the Environment, 2008a, 17, 149-159.

Weber O., Scholz R., Michalik G. Incorporating Sustainability Criteria into Credit Risk Management. Business Strategy \& the Environment,2008b.

Wood D. Corporate Social Performance revisited. Academy of Management Review, 1991, 16(4), 691-718.

Wright C. Setting Standards for Responsible Banking: Examining the Role of the International Finance Corporation in the Emergence of the Equator Principles, in Biermann F., Siebenhüner B. and Schreyrogg A. (eds.), International Organizations and Global Environmental Governance, 2007, London: Routledge.

Wright C., Rwabizambuga A. Institutional Pressures, Corporate Reputation and Voluntary Codes of Conduct: An Examination of the Equator Principles. Business and Society Review, 2006, 111(1), 89117. 